\title{
Do functional identity and divergence promote aboveground carbon differently in tropical semi-arid forests and savannas?
}

\author{
Sylvanus Mensah (iD) $1, \uparrow$ Fons van der Plas (D), 2,3 and Florent NoulèKoun (D) 4 \\ ${ }^{1}$ Laboratoire de Biomathématiques et d'Estimations Forestières, Faculté des Sciences Agronomiques, Université d'Abomey Calavi, \\ Cotonou, Benin \\ ${ }^{2}$ Plant Ecology and Nature Conservation Group, Wageningen University, PO Box 47, Wageningen, AA 6700 The Netherlands \\ ${ }^{3}$ Systematic Botany and Functional Biodiversity, Institute of Biology, Leipzig University, Johannisallee 21-23, Leipzig 04103 Germany \\ ${ }^{4}$ Department of Environmental Science and Ecological Engineering, Korea University, 145 Anamro, Seongbukgu, Seoul 02841 Korea
}

Citation: Mensah, S., F. van der Plas, and F. Noulèkoun. 2021. Do functional identity and divergence promote aboveground carbon differently in tropical semi-arid forests and savannas? Ecosphere 12(6):e03563. 10.1002/ecs2.3563

\begin{abstract}
Various studies have shown that plant species richness can promote ecosystem functions such as biomass storage. However, it is less well known whether this is mostly driven by the dominance of a few species and their associated traits (functional identity), or by complementarity among species that highly vary in their traits (functional diversity). The relative contribution of functional diversity and functional identity on biomass and carbon storage may in part depend on the type of functional traits that are considered, and on ecosystem type. Here, we used forest inventory data from West African semi-arid environments, and functional traits (wood density and tree maximum height) to examine the effects of functional trait identity (FI or community weighted mean; CWM) and diversity (FD or single functional divergence; FDvar) on aboveground carbon (AGC) storage in both forests and savannas. We fitted simple linear and structural equation models to test the direct and indirect effects of functional traits on AGC, while accounting for potential effects of vegetation stand structure such as stand density and basal area. When evaluated independently, CWM of tree maximum height and FDvar of wood density correlated positively with AGC, in both forests and savannas, whereas species richness was unrelated to AGC. However, structural equation models indicated different mechanisms by which these biodiversity components drove AGC in forests and savannas. In forests, species richness had an indirect, positive effect on AGC via basal area, but also an indirect, negative effect, through a reduction in CWM of maximum height. In savannas, species richness had a direct, negative effect on AGC, while both CWM of maximum height (through an increase in basal area) and FDvar of wood density had positive effects. Our study suggests that integrative models are crucial for understanding the effects of species richness, functional trait diversity, and identity on AGC across forests. Furthermore, our study shows that relationships between biodiversity and AGC differ among ecosystem types. In both forests and savannas, FI played an important role, as AGC was maximized in communities dominated by species with a high maximum height. However, only in savannas a high FD additionally promoted AGC.
\end{abstract}

Key words: community weighted mean; functional diversity; maximum plant height; semi-arid; tree biomass carbon.

Received 3 August 2020; revised 1 January 2021; accepted 14 January 2021; final version received 8 April 2021. Corresponding Editor: Jennifer M. Fraterrigo.

Copyright: (c) 2021 The Authors. This is an open access article under the terms of the Creative Commons Attribution License, which permits use, distribution and reproduction in any medium, provided the original work is properly cited.

† E-mail: sylvanus.m89@gmail.com

\section{INTRODUCTION}

Community ecologists have frequently used taxonomic or species diversity measures to draw inference on biodiversity effects on ecosystem functions. Across ecosystems, studies have reportedly shown that species richness or related taxonomic measures such as evenness or 
Shannon diversity have positive effects on biomass production (Vance-Chalcraft et al. 2010, Chisholm et al. 2013, Wu et al. 2015). However, species richness only accounts for the number of different species, but not necessarily for their functional redundancy or complementarity. As such, it may provide only limited understanding of functional mechanisms operating at the community level. From a mechanistic perspective, different species can be functionally equal and therefore would likely contribute equally to achieve the same function, while other species that are functionally dissimilar may complement each other in their contributions to ecosystem functioning (Connor et al. 2016, Mensah et al. 2020c).

There is mounting evidence that information on species functional traits therefore sheds more light on biodiversity effects on community biomass production than species richness (Díaz and Cabido 2001, Finegan et al. 2015, Mensah et al. 2016a, 2020c, Hao et al. 2018). Accordingly, two main community-level indicators of functional traits have been extensively considered: trait diversity (functional diversity) and trait identity (functional identity). Functional diversity (FD) is a measure of the variability of specific traits among species in an ecosystem, and it has been shown that it can have positive effects on ecosystem functions (Mensah et al. 2016a, Gross et al. 2017, Wen et al. 2019) and that it can mediate the positive effect of species richness on aboveground carbon (AGC; Mensah et al. 2016a, 2018b, 2020c). This may be in part because communities with more functionally different species ought to have greater resource-use complementarity and functioning compared to monoculture or communities containing functionally redundant species (Cadotte 2017, van der Plas 2019). As opposed to FD and niche complementarity, functional trait identity (FI), which describes which traits within a community are most dominant, and which is typically measured as the Community Weighted Mean (CWM) of a trait, can also have strong effects on ecosystem functions (Lin et al. 2016, Wasof et al. 2018). Typically, significant relationships between FI and ecosystem functioning are interpreted as providing evidence that species with certain traits provide more to ecosystem functioning than species with other traits.

ECOSPHERE *www.esajournals.org
Two critical points are emphasized in this study. The first is that while many studies have provided evidence that FD and FI measures underlie species diversity effects on biomass, the relative importance of their effects may vary with both the functional traits that are measured (Conti and Díaz 2013, Finegan et al. 2015) and with habitat types. For example, Conti and Díaz (2013) reported that unlike leaf traits, CWM and functional diversity of wood traits (height and wood density) best predicted aboveground standing biomass and productivity in semi-arid forests. Meanwhile, other studies found that CWM of specific leaf area and CWM of leaf nitrogen content are important for biomass increments and carbon storage and sequestration (Finegan et al. 2015, Mensah et al. $2018 b$ ). Further, in a study conducted in a natural evergreen broad-leaved forest in southeast China, Lin et al. (2016) found a negative association between CWM of wood density (WD) and AGC (see their Fig. 2a), which was also observed in natural Mistbelt forests in South Africa (Mensah et al. 2016a). In opposition to these findings, PradoJunior et al. (2016a) found that CWM of WD positively influenced aboveground biomass in dry tropical forests. These different findings suggest that functional trait effects are controlled by ecosystem type and environment, which govern plant plasticity, longevity, growth rate, and biomass retention. For instance, in semi-arid environments, some plants may benefit from a high longevity, even if this is associated with a slow growth, while pioneer species may profit more from a fast growth rate and high competitive abilities (Wright et al. 2004, Chave et al. 2009). As such, biomass and carbon storage may be driven either by the effects of ecological adaptations of conservative species (e.g., high wood density) or by the effects of fast-growing acquisitive species (e.g., high specific leaf area; Fortunel et al. 2014, Finegan et al. 2015, Prado-Junior et al. 2016a). Because species growth habits and resource use strategies vary across environment and habitat, there is a need for more studies to understand functional diversity and identity effects on biomass and carbon storage, particularly across ecosystems.

The second important issue is that many studies have not considered different directions of causality regarding relationships between species richness, FD and FI, and AGC, nor have they explicitly accounted for stand structural 
attributes as potential covariates of FD and FI. In many studies, the assumption is that FD and FI are driven by taxonomic diversity and that FI and FD underlie relationships between taxonomic diversity and carbon storage (Paquette and Messier 2011, Vilà et al. 2013, Hao et al. 2018). However, it is possible that FI and FD do not respond to variation in species richness, but drive variation in species richness. For example, in communities dominated by many tall trees (and which consequently have a high CWM of maximum height), interspecific competition for light may be intense, which can decrease species richness through competitive exclusion of weak competitors or shade intolerant species. In benign and productive environments, it is expected that a (few) strong competitor(s) will outcompete inferior ones, thereby resulting in low diversity (Carroll et al. 2011, Kim and Ohr 2020). As such, increasing FI values of traits related to competitive ability (e.g., tree maximum height) may lead to a reduced species richness (i.e., few dominant and other species that can tolerate low levels of light). On the other hand, because traits determine how an organism extracts resources from its environment (McGill et al. 2006), a high diversity in plant traits (i.e., increasing FD) would promote resource partitioning and species coexistence and thereby species richness, as species can reduce interspecific competition by using resources differently (Cadotte et al. 2011, Mensah et al. 2018b). Such alternative directions in the causality of relationships between species richness, FD and FI may also have consequences for the interpretation of relationships between species richness and AGC and may, for example, imply that observed correlations between species richness and AGC are not necessarily causal, but driven by FD or FI. Finally, AGC is expected to increase with stand structural attributes such as tree stock density and/or stand basal area (Mensah et al. 2016b, 2020a). However, as growth traits underpin stand vegetation structures, the effect of functional traits on AGC may also overlap with that of stand structural attributes, which if not accounted for, can obscure the magnitude of the influence of functional traits diversity.

Here, we focus on the vegetation in West Africa, largely underrepresented in the current literature on biodiversity and ecosystem functioning relationships (van der Plas 2019). The vegetation in West Africa is known to be remarkably diverse in terms of its physiognomy, from the Guinean littoral forests to the southern Sahel. Among its diverse forms, the forest-savanna mosaic lies within the semi-arid transitional zone between the Guinean littoral forests (in the south) and the Sudanian savannas (in the north). Using forest inventory data from a 10-ha sampled area, we investigated the effects of FI and FD on AGC in West African semi-arid forests and savannas. In particular, we had two specific objectives: (1) evaluate AGC response to individual effects of species richness, stand structure (stand density and basal area), FI (community weighted means of trait values), and FD (divergence of trait values) in both gallery forests and savannas; and (2) determine how species richness, FD, FI, and stand structures interact to maintain AGC. We tested four hypotheses: (1) FI and FD promote AGC in both forests and savannas after accounting for the effects of stand structure; (2a) species richness effects on AGC operate through non-mutually exclusive mediations by stand structure, FI and FD. Alternatively, we also tested whether (2b) FI would influence both species richness and AGC; and (2c) FD would increase species coexistence (and thus richness) and AGC through a greater complementarity in resource use.

\section{Materials and Methods}

\section{Study area and data}

We used forest inventory data from our previous study, which was carried out in the Bellefoungou Forest Reserve located in the SudanoGuinean transition zone in the northern part of the Republic of Benin. The forest reserve is situated between $1^{\circ} 42^{\prime} 00^{\prime \prime}$ and $1^{\circ} 45^{\prime} 00^{\prime \prime}$ E longitude and $9^{\circ} 46^{\prime} 40^{\prime \prime}$ and $9^{\circ} 49^{\prime} 00^{\prime \prime} \mathrm{N}$ latitude and is a complex of woodlands, gallery forests, savannas, and plantations, occurring at low elevation of $405 \mathrm{~m}$ asl. The climate is of Sudano-Guinean type, with average daily temperature of $28^{\circ} \mathrm{C}$ and annual rainfall of $1200 \mathrm{~mm}$. The rain occurs between April and October and has its peak between July and August. Additional information about the study area can be found in Mensah et al. (2020a).

We used a dataset from a 10 ha sampled area in West African semi-arid gallery forests 
(hereafter referred to as forests) and tree/shrub savannas (hereafter referred to as savannas) and collected using a stratified random sampling scheme. We sampled a total of 54 plots of 0.18 ha each across two vegetation types: forests (24 plots) and savannas (30 plots). For forest inventory, all individual trees with a diameter at breast height ( $\mathrm{dbh}) \geq 5 \mathrm{~cm}$ were measured for their $\mathrm{dbh}$ and total height and identified at the species and genus levels following the flora of Benin (Akoègninou et al. 2006). Plot-level species richness varied from 8 to 24 in forests and from 8 to 14 in savannas. As the study was conducted at a local scale, these plots were quite homogenous in terms of climate, soil, and topography within each vegetation type. Nonetheless, it is important to mention that gallery forests, as opposed to tree/shrub savannas, have better plant growing conditions (e.g., higher water and nutrient availability) induced by the presence of running surface water. These growing conditions make it possible for a wide range of species to reach larger diameter size in the forests, while comparatively unfavorable conditions of drought and low soil humidity in savannas, in addition to higher evapotranspiration caused by their open vegetation canopy, tend to limit the prevalence of large-size trees (Mensah et al. 2020a). Additional information on stand structure (tree density and basal area) and AGC are summarized in Appendix S1: Table S1.

\section{Quantification of stand structure and aboveground biomass carbon}

We calculated plot-level species richness, tree density, and basal area, using the abundance and tree diameter data for each vegetation type. We applied the multispecies allometric biomass equation developed by Chave et al. (2014) for all individual trees present in the plots to determine each tree biomass. The formula for the multispecies allometric biomass equation is as follows: $\mathrm{AGB}=0.0673 \times\left(\rho \times \mathrm{DBH}^{2} \times H\right)^{0.976}$, where AGB is the aboveground tree biomass $(\mathrm{kg}), \rho$ the species-specific wood density $\left(\mathrm{g} / \mathrm{cm}^{3}\right)$, DBH the diameter at breast height $(\mathrm{cm})$, and $H$ the total height $(\mathrm{m})$ measured for each individual tree. The plot-level aboveground tree carbon (AGC) was then calculated by summing up the aboveground biomass of each individual tree measured within a plot and applying a carbon fraction of 0.5 (Lung and Espira 2015, Mensah et al. 2020a).

While data on wood density were used in our calculation of AGC, due to the non-linearities in our equations, observed relationships between FD or FI values of wood density and AGC are likely primarily driven by ecological processes rather than by methodological choices. Furthermore, while the quantification of AGC directly depends on stand structure (tree density and basal area), given that we were primarily interested in the consequences of tree diversity and functional attributes on AGC, we did not deem this as problematic.

\section{Quantification of functional trait diversity and identity metrics}

In this study, we investigated FI and FD based on two functional traits that are relevant to tree growth and biomass production: specific wood density and tree maximum height. Species wood density reflects the strategy of a species to allocate resources into fast growth or slow growth, such that low wood density species exhibit fast growth as opposed to denser wood species which have slow-growing habit. Maximum height reflects the species longevity and life-history strategy as well as its potential for light capture. Data on speciesspecific wood density were obtained from our previous investigation in the study area (Mensah et al. 2020a). Tree maximum height was determined for each species from empirical measurements at the study site. To quantify functional trait diversity, we computed for each plot two single functional trait variability metrics: FDvar (Mason et al. 2003) for wood density and tree maximum height. For functional identity metrics, we computed the plot-level CWM for each functional trait (Garnier et al. 2004), as the mean of each species trait value weighted by the relative abundance of the species. Both functional trait diversity and identity metrics were calculated using the values of the species functional traits in the FD package (Laliberté et al. 2015).

\section{Statistical analyses}

All statistical analyses were performed in the $\mathrm{R}$ statistical software package, version 4.0.0 (R Core Team 2020). We built Pearson-based correlation matrix heat maps to explore the correlation between all the variables used in this study 
(Appendix S1: Fig. S1). In addition, we fitted multiple linear models with species richness, stand structure (stand density and basal area), FDvar, and CWM for wood density and tree maximum height as predictors of AGC. We then tested for multicollinearity by means of variance inflation factor (VIF), with values higher than 5 indicating that there is multicollinearity (Appendix S1: Table S2). We found overall weak correlations between most predictors (Appendix S1: Fig. S1), with all VIF values being lower than 5 for both forests and savannas (see Appendix S1: Table S2). Shapiro-Wilks statistics also indicate that the residuals of the multiple linear models are normally distributed $(W=0.96, P=0.474$ in forests and $W=0.97, P=0.550$ in savannas).

Our first objective was to evaluate the response of AGC to individual effects of species richness, vegetation stand structure (stand density and basal area), functional identity (CWM of trait values), and functional diversity (divergence of trait values) in both forests and savannas. We did so by testing for individual effects of stand structures and functional trait diversity and identity on AGC using simple linear models. In particular, we explored the bivariate relationships of AGC with species richness, stand density, basal area and functional diversity and functional identity metrics as defined above (Appendix S1: Table S3). All variables were standardized to a mean of zero and unit variance to allow for equal weight so that their relative effects could be compared. The standardized slope coefficients of these bivariate relationships were plotted for better visualization of the effects. One aspect that we considered in this study was that stand structural attributes can mediate the effects of functional diversity and identity on AGC across ecosystems. As vegetation structure (particularly stand basal area) is often related to biomass and productivity, it was important to account for the possible confounding effects of vegetation stand structure when assessing functional identity and diversity effects. To do so, we removed the potential effect of stand density and basal area on AGC with a simple linear regression (using the standardized plot-level values for each vegetation type) and repeated the simple linear models with the residuals of the AGC against stand structure, replacing AGC as the response variable (Appendix S1: Table S4).
Our second objective was to assess the direct and indirect effects of species richness, as well as how stand structural attributes, functional diversity and identity interact to maintain AGC. With a separate dataset for forests and savannas, we fitted Structural Equation models (SEMs) to test whether and how (1) stand structural attributes (stand density and/or basal area) and functional identity and diversity influence AGC (Fig. 1); and (2) species richness influences AGC directly or indirectly through its influence on vegetation stand structural attributes and functional trait metrics (Fig. 1). We acknowledge that environmental factors (climate, soil, and topography) could also influence AGC, but these factors would have minor impacts here due to the scale of the study and the homogeneity in our plots. The SEMs were fitted separately for forests and savannas. We started by fitting a full mediation SEM (i.e., Fig. 1; all paths minus the direct path between species richness and AGC; see also Appendix S1: Table S5, Fig. S2) for each vegetation type. Using the path modification indices, we then improved the model fit, by performing a step-by-step inclusion of additional significant paths leading to partial mediation SEMs. We compared the full mediation and partial mediation models based on the goodness-of-fit statistics and AIC values (Grace and Bollen 2005). When fitting these SEMs, we only considered stand structure and functional diversity and identity measures that significantly influenced AGC (Fig. 2).

While functional traits can mediate species richness effects on AGC, the dominance of species with certain traits can have a negative feedback on stand species richness through competitive selection effects, whereas trait diversity can promote biomass carbon stock by facilitating a diversified community assemblage and greater complementarity in resource use. Thus, for each vegetation type, we further constructed two SEMs testing separately for functional identity and diversity feedbacks on species richness, while accounting for stand structure (both tree density and basal area; see Appendix S1: Table S6, Fig. S3). All SEMs were fitted using the lavaan package (Rosseel 2012). We evaluated the overall fit of the models to the data using chi-square $(P>0.05$ indicating good fit to the data), the comparative fit index (CFI), the goodness-of-fit index (GFI), and the standardized root mean square residual (SRMR). 




Fig. 1. A conceptual model testing the interplay between functional diversity, functional identity and stand structures and their influence on aboveground carbon (AGC). Paths $a, b$, and c tested the indirect influence of species richness through functional identity whereas paths $d, e$, and $f$ tested the indirect influence of species richness through functional diversity. We also tested how AGC is influenced directly by species richness (path $h$ ), and indirectly through stand structure (paths g and i). Abbreviations are Spp Rich, species richness; CWM, community weighted mean; FDvar, functional diversity; Stand Struct., stand structures.



Fig. 2. Slope coefficients of the bivariate relationships of stand aboveground carbon (AGC) with stand structure, functional trait diversity, and identity measures. Abbreviations are CWM, community weighted mean; FDvar, functional diversity; $\mathrm{Hm}$, tree maximum height; WD, wood density; TreeG, basal area; TreeN, tree density; Spp, species richness.

\section{RESULTS}

\section{Bivariate relationships between biodiversity indicators and AGC}

AGC was consistently and positively associated with stand basal area, CWM of maximum height, and FDvar of wood density in both forests and savannas (Fig. 2; Appendix S1: Table S3). CWM of wood density and FDvar of maximum height did not have any significant influence on AGC. Similarly, tree density and species richness had no significant effects on AGC (Fig. 2). Together, tree density and basal area explained $70.63 \%$ of the variation of AGC in forests and $92 \%$ in savannas. When accounting for both the stand density and basal area effects on AGC, we found that only CWM of maximum height promoted AGC in forests while only FDvar of wood density had a marginally positive effect on AGC in savannas (Appendix S1: Table S4).

\section{Structural equation models}

The initial full mediation SEM did not fit well with the forest plots data $(P=0.011$; Appendix S1: Table S5), unlike that of savannas $(P=0.066 ; \quad$ Appendix S1: Table S5), which 
showed significant positive effects of CWM of maximum height on basal area and of basal area on AGC $(P<0.05 ;$ Appendix S1: Table S5, Fig. S2). Nevertheless, both full mediation SEMs had higher AIC compared to the improved partial mediation SEMs, which showed better fits in forests and savannas, with $P>0.05$ and $\mathrm{CFI}$, GFI, and SRMR values all within the acceptable range (Fig. 3).

In forests, CWM of maximum height had a positive direct effect on AGC $(\beta=0.38$, $P<0.001$; Table 1 ; Fig. $3 \mathrm{~A})$ and a positive indirect effect through basal area $(\beta=$ $0.50 \times 0.73=0.37, \quad P=0.004 ; \quad$ Fig. $3 \mathrm{~A})$. Thus, effects of CWM of maximum height operated partially through basal area, and directly to AGC, with a total standardized effect (i.e., direct plus indirect) of $\beta=0.75(P<0.001)$. Meanwhile, CWM of maximum height significantly decreased with species richness $(\beta=-0.42$, $P=0.010$; Table 1; Fig. 3A), which also increased stand basal area $(\beta=0.36$; Fig. $3 \mathrm{~A}$; Table 1$)$. Therefore, species richness had two indirect effects on AGC: one positive effect via basal area $(\beta=0.36 \times 0.73=0.26$; Table 1$)$, and a negative effect via CWM of maximum height. Unlike CWM of maximum height, FDvar of wood density did not significantly influence basal area and AGC in forests $(P=0.268$; Fig. 3A; Table 1$)$ and was not correlated with species richness $(\beta=0.05, P=0.794)$. These results indicate that AGC stocks in forests were shaped by three main variables: basal area (direct effect), CWM of maximum height via a direct effect and an indirect effect through an increase on basal area, and species richness via mediation through basal area and CWM of maximum height. Further, when we compared the direct, indirect, and total effect of functional identity and diversity, we found that only CWM of maximum height had a significant and positive total effect on AGC in forests (Fig. 3B).

A different pattern was observed in savannas (Table 1; Fig. 3C); in addition to the positive indirect effect of CWM of maximum height on AGC via basal area $(\beta=0.50 \times 0.88=0.44$, $P<0.001$; Fig. $3 C)$, FDvar of wood density promoted AGC directly $(\beta=0.11, P=0.043)$. Further, both CWM of maximum height and FDvar of wood density were not significantly influenced by species richness $(P>0.05)$, which was found to influence AGC through a negative direct effect $(\beta=-0.11, P=0.010$; Table 1$)$. Thus, in savannas, both CWM of maximum height and FDvar of wood density promoted AGC (Fig. 3D).

The alternative SEM (Appendix S1: Table S6, Fig. S3) testing for functional identity and diversity feedback on species richness, while accounting for stand structure also confirmed that CWM of maximum height and FDvar of wood density were strongly correlated with basal area in both forests and savannas (Appendix S1: Fig. S3). However, in forests, CWM of maximum height had a significant negative feedback on species richness while influencing positively AGC (Appendix S1: Fig. S3). FDvar of wood density did not show any significant association with AGC or species richness in forests, in accordance with the SEM in Fig 3A. In savannas, FDvar of wod density and not CWM of maximum height exhibited a positive direct effect on AGC (Appendix S1: Fig. S3). It is important to note that the conceptual SEMs where species richness influenced FD and FI (and not vice versa) (Fig. 3) were the best fitting models, as the alternative SEMs showed higher values of AIC (Appendix S1: Table S6), indicating that richness drove FI and FD.

Overall, the results showed that stand basal area correlated strongly with both CWM of maximum height and FDvar of wood density, with only the former substantially increasing AGC in both forests and savannas.

\section{DISCUSSION}

Various studies reported that species richness effects on aboveground biomass or productivity are mediated by stand basal area and/or tree density in natural forests (Chisholm et al. 2013, Vilà et al. 2013). For instance, the effect of species richness on biomass or productivity is attributable to local variation in stem density at small spatial scales (Chisholm et al. 2013), or to higher species richness promoting stocking density (Mensah et al. 2020b) or basal area (Vilà et al. 2013). From a functional perspective, the effect of stand structural attributes could potentially obscure that of species richness or functional trait diversity on stand AGC because functional traits contribute to individual tree growth. In this 
A - Forest

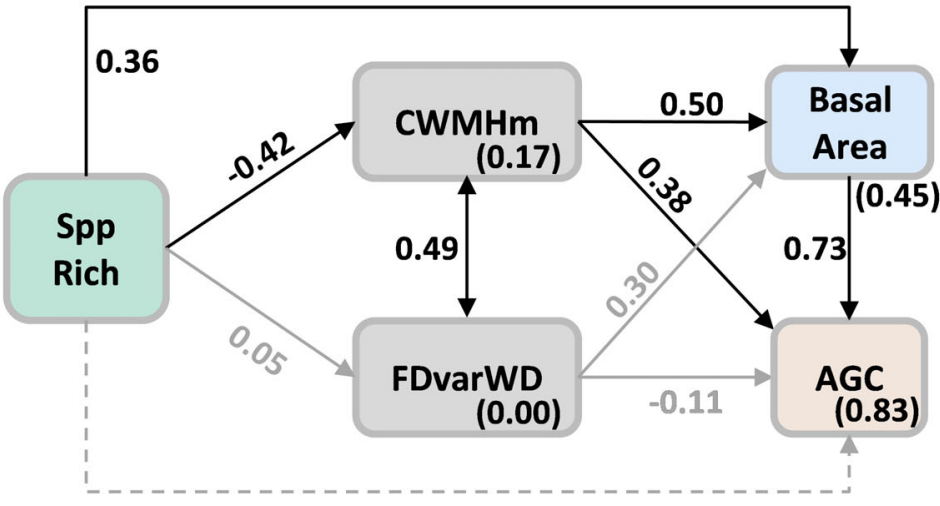

$\mathrm{df}=1 ; P=0.110 ; \mathrm{CFI}=0.97 ; \mathrm{GFI}=0.95 ; \mathrm{SRMR}=0.03 ; \mathrm{AIC}=226$
B - Forest

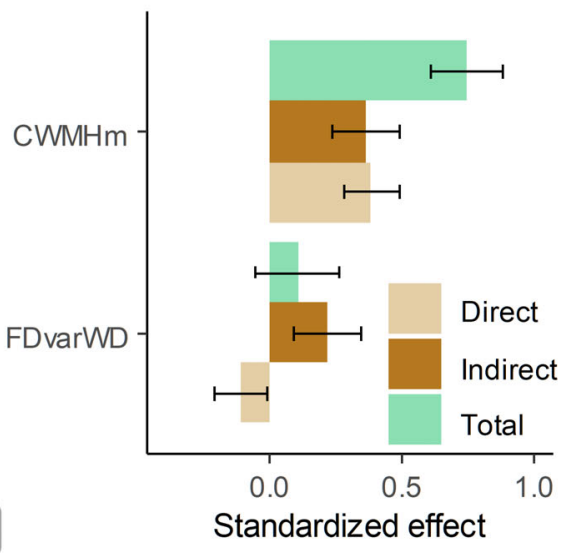

D - Savanna

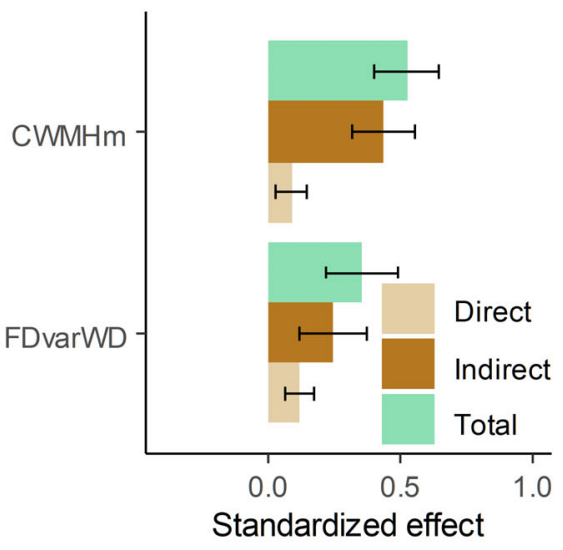

$\mathrm{df}=1 ; P=0.318 ; \mathrm{CFI}=1.00 ; \mathrm{GFI}=0.98 ; \mathrm{SRMR}=0.06 ; \mathrm{AIC}=269$

Fig. 3. Summary of the final partial mediation SEMs paths relating species richness and aboveground carbon (AGC) through functional identity (CWMHm), functional diversity (FDvarWD), and stand structure (basal area) in forests (A) and savannas (C). In (B) and (D), we showed the direct, indirect, and total effects of functional identity and diversity on AGC for forests and savannas, respectively. The values without parentheses on the SEM graph are the standardized path coefficients. The values with parentheses are the coefficients of determination ( $R$ square), shown for the dependent variables. The single-pointed arrows are the causal paths. The double-pointed arrows are non-causal correlations. The dotted lines are the non-significant paths that were excluded to fit a nonsaturated partial mediation SEM. The black arrows indicate significant effects and the gray arrows non-significant effects. Significance of standardized path coefficients is also given in Table 1. Spp Rich, species richness; CWM, community weighted mean; FDvar, functional diversity; Hm, tree maximum height; WD, wood density; AGC, aboveground carbon; $\mathrm{df}$, degree of freedom; $P$, probability associated with chi-square test $(P>0.05$ indicates absence of significant discrepancy between the data and the model); CFI, comparative fit index; GFI, goodness-of-fit index; SRMR, standardized root mean residual; AIC, Akaike information criteria.

study, when evaluated independently, both functional identity of tree maximum height (CWM of maximum height) and divergence of wood density (FDvar of wood density) had positive effects on AGC in both forests and savannas. However, when the effects of stand structural attributes were accounted for, only CWM of maximum height influenced AGC in forests and only FDvar 
Table 1. Summary of fit statistics and significance of the final SEM paths relating species richness and aboveground carbon through functional identity (CWMHm), functional diversity (FDvarWD) and stand structure (basal area) in forests and savannas.

\begin{tabular}{lccccc}
\hline \hline Predictor & Response variable & Est.std. & SE & Z & $P$ \\
\hline Forests & & & & & \\
Species richness & CWMHm & -0.42 & 0.16 & -2.57 & $\mathbf{0 . 0 1 0}$ \\
Species richness & FDvarWD & 0.05 & 0.20 & 0.26 & 0.794 \\
Species richness & Basal area & 0.36 & 0.16 & 2.17 & $\mathbf{0 . 0 3 0}$ \\
Species richness & AGC & $\ldots$ & $\ldots$ & $\ldots$ & $\ldots$ \\
CWMHm & Basal area & 0.50 & 0.18 & 2.79 & $\mathbf{0 . 0 0 5}$ \\
CWMHm & AGC & 0.38 & 0.10 & 3.67 & $<\mathbf{0 . 0 0 1}$ \\
FDvarWD & Basal area & 0.30 & 0.17 & 1.75 & 0.081 \\
FDvarWD & AGC & -0.11 & 0.10 & -1.11 & 0.268 \\
Basal area & AGC & 0.73 & 0.09 & 7.49 & $<\mathbf{0 . 0 0 1}$ \\
Savannas & & & & & \\
Species richness & CWMHm & -0.16 & 0.18 & -0.88 & 0.379 \\
Species richness & FDvarWD & 0.13 & 0.18 & 0.70 & 0.484 \\
Species richness & Basal area & 0.05 & 0.15 & 0.30 & 0.764 \\
Species richness & AGC & -0.11 & 0.05 & -2.14 & $\mathbf{0 . 0 3 2}$ \\
CWMHm & Basal area & 0.50 & 0.14 & 3.69 & $<\mathbf{0 . 0 0 1}$ \\
CWMHm & AGC & 0.09 & 0.06 & 1.40 & 0.162 \\
FDvarWD & Basal area & 0.27 & 0.15 & 1.83 & 0.067 \\
FDvarWD & AGC & 0.11 & 0.06 & 2.02 & $\mathbf{0 . 0 4 3}$ \\
Basal area & AGC & 0.88 & 0.05 & 17.64 & $<\mathbf{0 . 0 0 1}$ \\
\hline
\end{tabular}

Notes: $P<0.05$ appear in boldface. Est.std., standardized estimates; SE, standard error; CWM, community weighted mean; FDvar, functional diversity; Hm, tree maximum height; WD, wood density; AGC, aboveground carbon; ..., indicates the nonsignificant path that was excluded to fit a non-saturated partial mediation SEM.

of wood density marginally influenced AGC in savannas (Appendix S1: Table S4). While these results support our first hypothesis on the consistent effects of FD and FI on AGC, they further reveal that the magnitude of these effects is reduced when accounting for stand structural attributes. The smaller positive effect of FD and FI on AGC after accounting for stand structural attributes suggests that FD and FI may not necessary influence AGC directly and may have an indirect effect through stand basal area and tree density (Chisholm et al. 2013, Vilà et al. 2013, Mensah et al. 2020b). This result further suggests that a failure to account for vegetation stand structure across ecosystems can potentially obscure the relative importance of FD and FI of wood traits as drivers of AGC storage. Thus, integrative models could provide a significant advantage in understanding the effects of FD, FI, and stand structural attributes on AGC across forests.

Consistent with the results of previous studies, the more integrative SEMs in our study indicate that carbon stock was positively driven by functional identity of tree maximum height in both forests (through direct and indirect effects) and savannas (through an indirect effect via basal area). Recently, Lin et al. (2016) and Dimobe et al. (2019) showed that AGC increased markedly with CWM of plant height in a nature reserve in Asia and in West African semi-arid ecosystems, respectively. Similarly, CWM of maximum plant height reportedly had positive effects on aboveground biomass and carbon in Mistbelt forests (Mensah et al. 2016a) and in tropical and semi-arid forests (Conti and Díaz 2013, Finegan et al. 2015). Furthermore, Cadotte (2017) also reported that the CWM of plant height was an important predictor of biomass in established plant assemblages. One explanation for tree maximum height being a potential trait with predictive power for AGC is that it defines the longterm forest vertical stratification, which plays a crucial role in light interception and light use efficiency by plants in forest ecosystems (Poorter et al. 2006, Yachi and Loreau, 2007, Morin et al. 2011). Further, tree height is an important variable that could explain the variation in growth 
and biomass because species and individuals with the same diameter often exhibit different tree heights and would probably have different allometries (Mensah et al. 2018a). This insight is also evidenced by the direct effect of CWM of maximum height on AGC and its indirect effect which operated through basal area (Fig. 3). Tree maximum height often correlates strongly with medium and large stand diameters, which reportedly reflect stand dominance patterns and represent a high proportion of basal area and AGC (Lutz et al., 2018, Mensah et al. 2020a). Our finding that high community weighted mean values of tree height promoted AGC is in line with the idea that dominant species and their associated traits have greatest influence on ecosystem functioning (Carroll et al. 2011, Mensah et al. 2016a). Specifically, a high CWM of maximum height indicates the dominance of tall species, which are likely superior in their ability to compete for light in forest ecosystems (Carroll et al. 2011, Cadotte 2017).

The SEM fitted to the data from savannas indicated that, in addition to the positive CWMHm effect, FDvar of wood density also promoted AGC directly (Fig. 3C). This shows that unlike the pattern observed in the forests, diversity in wood density additionally promoted AGC in savannas, supporting the niche complementarity hypothesis. The shift in the effect of FDvar of wood density from neutral in forests to positive in savannas was a striking finding from the integrative SEMs (Fig. 3), which is attributable to the conditions that maintain forest and savanna vegetation types in the study region. In the gallery forests, favorable conditions (e.g., high water and nutrient availability) due to the presence of running surface water support a wider range of species, including some fast-growing species characterized by acquisitive traits, and capable of reaching large diameter and high height in the forests (Fortunel et al. 2014, Jucker et al. 2016). This explains the greater direct and indirect effects of CWMHm on AGC in the forests, as compared to savannas. In contrast, unfavorable conditions of drought and low humidity in savannas' soils, added to higher evapotranspiration due to their open canopies, may lead to the dominance of species with conservative resource-use strategies to achieve survival and biomass retention in resource-limited environments (Chave et al. 2009, Fortunel et al. 2014). Such conservative strategies occur through species-specific investments in conductive tissues, which could increase variability in species wood density. Moreover, wood density reflects a trade-off between fast growth rate (associated with low wood density) and low mortality (associated with high wood density; Chave et al. 2009, Philipson et al. 2014, Prado-junior et al. 2016b). Therefore, our result implies that vegetation codominated by species with fast growth rates and low mortality (thereby spanning the whole gradient of wood density) maximize AGC, possibly because having both strategies allows for the maximization of biomass production in both good (high rainfall, few disturbances) and bad (low rainfall, more disturbances) years.

The smaller effect of CWM of maximum height in savannas (as compared to the patterns in forests) could be attributed to (1) the generally smaller density of large-size and often tall trees, and relatively higher abundance of small-size trees, as compared to forests, and (2) the scattered pattern of trees in savannas, resulting in relatively lower stocking density per ha and subsequent less competition for light in these habitats compared to forests. These small-size trees also often occur within a considerable species pool with varying growth habit and stem structure, which also mechanistically explains the positive association between AGC and functional divergence in wood density in savannas. Previous studies have inferred on niche complementarity as mechanism driving ecosystem function by considering multiple traits functional diversity metrics (Cadotte 2017, Huang et al. 2020). For instance, Cadotte (2017) showed that niche differentiation and complementarity effects were only explained by multi-trait combinations. Our study, however, shows that the niche complementarity can also be explained by a single trait, as evidenced by the significant effect of divergence in wood density in savannas (Fig. 3; Appendix S1: Fig. S3).

Finally, we also found that species richness did not directly influence carbon stocks, but had an indirect effect by significantly reducing CWM of maximum height in forests. Importantly, model comparisons showed that richness influenced carbon stocks through its effects on FD and FI, and not vice versa. Thus, while a high species richness is important for maximizing AGC in 
both forests and savannas, its effects were mostly indirect and operated through changes in FI (in both forests and savannas) and FI (in savannas only).

\section{CONCLUSION}

This study examined the relationships between aboveground carbon, species richness, functional diversity, and identity measures of wood density and maximum height in forests and savannas, while accounting for the potential vegetation stand correlates such as tree density and basal area. We found that basal area correlated strongly with both CWM of maximum height and FDvar of wood density, with the former substantially increasing AGC in forests and both promoting AGC differently in savannas. Importantly, the study shows that integrative models are crucial for understanding the effects of species richness, functional trait diversity, and identity on AGC across forests and savannas and that different mechanisms drive the effects of biodiversity components on AGC in forests and savannas. For instance, the finding that AGC stock correlates positively with CWM of maximum height in forests and savannas suggests that AGC is maximized in communities dominated by species with a high maximum height. On the other hand, the additional effect of FDvar of wood density on AGC in savannas supports the mechanism of niche differentiation across the wood density trait space, as a driver of community tree growth and biomass carbon. Our findings therefore imply that the influence of functional diversity and identity vary with functional traits, and across ecosystems, possibly because of different species growth habit and dynamic.

\section{ACKNOWLEDGMENTS}

The authors are grateful to the colleagues at the Laboratoire de Biomathématiques et d'Estimations Forestières, Université d'Abomey-Calavi, for assisting with data collection. SM would like to thank the African Forest Forum (AFF) through the Research Grant on Land Use, Land Use Change and Forestry Linked to Climate Change. FN acknowledges the support provided by the BK21 PLUS Program (Brain Korea 21 Program for Leading Universities and Students) and the National Research Foundation (NRF) of Korea, which allowed fruitful international collaboration.

\section{Literature Cited}

Akoègninou, A., W. J. van der Burg, and L. J. G. van der Maesen. 2006. Flore analytique du Bénin. Backhuys Publishers, Leiden, The Netherlands.

Cadotte, M. W. 2017. Functional traits explain ecosystem function through opposing mechanisms. Ecology Letters 20:989-996.

Cadotte, M. W., K. Carscadden, and N. Mirotchnick. 2011. Beyond species: functional diversity and the maintenance of ecological processes and services. Journal of Applied Ecology 48:1079-1087.

Carroll, I. T., B. J. Cardinale, and R. M. Nisbet. 2011. Niche and fitness differences relate the maintenance of diversity to ecosystem function. Ecology 92:1157-1165.

Chave, J., et al. 2014. Improved allometric models to estimate the aboveground biomass of tropical trees. Global Change Biology 20:3177-3190.

Chave, J., D. Coomes, S. Jansen, S. L. Lewis, N. G. Swenson, and A. E. Zanne. 2009. Towards a worldwide wood economics spectrum. Ecology Letters 12:351-366.

Chisholm, R. A., et al. 2013. Scale-dependent relationships between tree species richness and ecosystem function in forests. Journal of Ecology 101:1214 1224.

Connor, M. I. O., et al. 2016. A general biodiversity function relationship is mediated by trophic level. Oikos 126:18-31.

Conti, G., and S. Díaz. 2013. Plant functional diversity and carbon storage - an empirical test in semi-arid forest ecosystems. Journal of Ecology 101:18-28.

Díaz, S., and M. Cabido. 2001. Vive la différence: plant functional diversity matters to ecosystem processes. Trends in Ecology \& Evolution 16:646-655.

Dimobe, K., S. Kuyah, Z. Dabré, A. Ouédraogo, and A. Thiombiano. 2019. Diversity-carbon stock relationship across vegetation types in W National park in Burkina Faso. Forest Ecology and Management 438:243-254.

Finegan, B., et al. 2015. Does functional trait diversity predict above-ground biomass and productivity of tropical forests? Testing three alternative hypotheses. Journal of Ecology 103:191-201.

Fortunel, C., C. E. T. Paine, P. V. A. Fine, N. J. B. Kraft, and C. Baraloto. 2014. Environmental factors predict community functional composition in Amazonian forests. Journal of Ecology 102:145-155.

Garnier, E., et al. 2004. Plant functional markers capture ecosystem properties during secondary succession. Ecology 85:2630-2637. 
Grace, J. B., and K. A. Bollen. 2005. Interpreting the results from multiple regression and structural equation models. Bulletin of the Ecological Society of America 86:283-295.

Gross, N., Y. Le Bagousse-Pinguet, P. Liancourt, M. Berdugo, N. J. Gotelli, and F. T. Maestre. 2017. Functional trait diversity maximizes ecosystem multifunctionality. Nature Ecology and Evolution $1: 132$.

Hao, M. H., C. Zhang, X. Zhao, and K. von Gadow. 2018. Functional and phylogenetic diversity determine woody productivity in a temperate forest. Ecology and Evolution 8:2395-2406.

Huang, M., X. Liu, M. W. Cadotte, and S. Zhou. 2020. Functional and phylogenetic diversity explain different components of diversity effects on biomass production. Oikos 129:1185-1195.

Jucker, T., A. C. Sanchez, J. A. Lindsell, H. D. Allen, G. S. Amable, and D. A. Coomes. 2016. Drivers of aboveground wood production in a lowland tropical forest of West Africa: teasing apart the roles of tree density, tree diversity, soil phosphorus, and historical logging. Ecology and Evolution 6:4004-4017.

Kim, D., and S. Ohr. 2020. Coexistence of plant species under harsh environmental conditions: an evaluation of niche differentiation and stochasticity along salt marsh creeks. Journal of Ecology and Environment $44: 19$.

Laliberté, E., P. Legendre, and B. Shipley. 2015. Measuring functional diversity (FD) from multiple traits, and other tools for functional ecology. $\mathrm{R}$ package version 1.0-12. https://cran.r-project.org/ web/packages/FD/index.html

Lin, D., K. J. Anderson-teixeira, J. Lai, X. Mi, H. Ren, and K. Ma. 2016. Traits of dominant tree species predict local scale variation in forest aboveground and topsoil carbon stocks. Plant and Soil 409:435446.

Lung, M., and A. Espira. 2015. The influence of stand variables and human use on biomass and carbon stocks of a transitional African forest: implications for forest carbon projects. Forest Ecology and Management 351:36-46.

Lutz, J. A., et al. 2018. Global importance of largediameter trees. Global Ecology and Biogeography 27:849-864.

Mason, N. W. H., K. MacGillivray, J. B. Steel, and J. B. Wilson. 2003. An index of functional diversity. Journal of Vegetation Science 14:571-578.

McGill, B. J., B. J. Enquist, E. Weiher, and M. Westoby. 2006. Rebuilding community ecology from functional traits. Trends in Ecology and Evolution 21:178-185.

Mensah, S., F. Noulèkoun, and E. E. Ago. $2020 a$. Aboveground tree carbon stocks in West African semi-arid ecosystems: dominance patterns, size class allocation and structural drivers. Global Ecology and Conservation 24:e01331.

Mensah, S., V. K. Salako, and T. Seifert. 2020b. Structural complexity and large-sized trees explain shifting species richness and carbon relationship across vegetation types. Functional Ecology 34:1731-1745.

Mensah, S., K. Valère, A. Assogbadjo, R. Glèlè, B. Sinsin, and T. Seifert. 2020c. Functional trait diversity is a stronger predictor of multifunctionality than dominance : evidence from an Afromontane forest in South Africa. Ecological Indicators 115:106415.

Mensah, S., O. L. Pienaar, A. Kunneke, B. du Toit, A. Seydack, E. Uhl, H. Pretzsch, and T. Seifert. 2018a. Height - Diameter allometry in South Africa's indigenous high forests: assessing generic models performance and function forms. Forest Ecology and Management 410:1-11.

Mensah, S., B. du Toit, and T. Seifert. 2018b. Diversitybiomass relationship across forest layers: implications for niche complementarity and selection effects. Oecologia 187:783-795.

Mensah, S., R. Veldtman, A. E. Assogbadjo, R. Glèlè Kakaï, and T. Seifert. 2016a. Tree species diversity promotes aboveground carbon storage through functional diversity and functional dominance. Ecology and Evolution 6:7546-7557.

Mensah, S., R. Veldtman, B. du Toit, R. Glèlè Kakaï, and T. Seifert. 2016b. Aboveground biomass and carbon in a South African Mistbelt forest and the relationships with tree species diversity and forest structures. Forests 7:79.

Morin, X., L. Fahse, M. Scherer-Lorenzen, and H. Bugmann. 2011. Tree species richness promotes productivity in temperate forests through strong complementarity between species. Ecology Letters 14:1211-1219.

Paquette, A., and C. Messier. 2011. The effect of biodiversity on tree productivity: from temperate to boreal forests. Global Ecology and Biogeography 20:170-180.

Philipson, C. D., D. H. Dent, M. J. O’Brien, J. Chamagne, D. Dzulkifli, R. Nilus, S. Philips, G. Reynolds, P. Saner, and A. Hector. 2014. A trait-based tradeoff between growth and mortality: evidence from 15 tropical tree species using size-specific relative growth rates. Ecology and Evolution 4:3675-3688.

Poorter, L., L. Bongers, and F. Bongers. 2006. Architecture of 54 moist-forest tree species: traits, tradeoffs, and functional groups. Ecology 87:1289-1301.

Prado-junior, J. A., I. Schiavini, V. S. Vale, C. S. Arantes, M. T. Van Der Sande, M. Lohbeck, and L. Poorter. 2016a. Conservative species drive biomass 
productivity in tropical dry forests. Journal of Ecology 104:817-827.

Prado-Junior, J. A., I. Schiavini, V. S. Vale, D. Raymundo, S. F. Lopes, and L. Poorter. 2016b. Functional traits shape size-dependent growth and mortality rates of dry forest tree species. Journal of Plant Ecology 10:895-906.

R Core Team. 2020. R: a language and environment for statistical computing. R Foundation for Statistical Computing, Vienna, Austria.

Rosseel, Y. 2012. lavaan: an R package for structural equation modeling. Journal of Statistical Software 48:1-36.

van der Plas, F. 2019. Biodiversity and ecosystem functioning in naturally assembled communities. Biological Reviews 94:1220-1245.

Vance-Chalcraft, H. D., M. R. Willig, S. B. Cox, A. E. Lugo, and F. N. Scatena. 2010. Relationship Between Aboveground Biomass and Multiple Measures of Biodiversity in Subtropical Forest of Puerto Rico. Biotropica 42:290-299.

Vilà, M., A. Carrillo-Gavilán, J. Vayreda, H. Bugmann, J. Fridman, W. Grodzki, J. Haase, G. Kunstler, M. J.
Schelhaas, and A. Trasobares. 2013. Disentangling Biodiversity and Climatic Determinants of Wood Production. PLOS ONE 8:e53530.

Wasof, S., et al. 2018. Dominance of individual plant species is more important than diversity in explaining plant biomass in the forest understorey. Journal of Vegetation Science 29:521-531.

Wen, Z., H. Zheng, J. R. Smith, H. Zhao, L. Liu, and Z. Ouyang. 2019. Functional diversity overrides community-weighted mean traits in liking landuse intensity to hydrological ecosystem services. Science of the Total Environment 682:583-590.

Wright, I. J., et al. 2004. The worldwide leaf economics spectrum. Nature 428:821-827.

Wu, X., X. Wang, Z. Tang, Z. Shen, C. Zheng, X. $X i a$, and J. Fang. 2015. The relationship between species richness and biomass changes from boreal to subtropical forests in China. Ecography 38:602613.

Yachi, S., and M. Loreau. 2007. Does complementary resource use enhance ecosystem functioning? A model of light competition in plant communities. Ecology Letters 10:54-62.

\section{SUPPORTING INFORMATION}

Additional Supporting Information may be found online at: http://onlinelibrary.wiley.com/doi/10.1002/ecs2. 3563/full 